TABLE VI.

\begin{tabular}{ccccc}
\hline \hline $\begin{array}{c}\text { Rim } \\
\text { shape }\end{array}$ & $\begin{array}{c}\text { Observed } \\
R_{\boldsymbol{c}}\end{array}$ & \multicolumn{2}{c}{ Predicted thickness } & Observed \\
\hline$G$ & $4 \times 10^{17} \mathrm{~cm}$ & $0.16 R_{\boldsymbol{c}}$ & 0.022 & 0.023 \\
4 & 5 & 0.16 & 0.027 & 0.034 \\
3 & 7.5 & 0.15 & 0.036 & 0.034 \\
2 & 10.5 & 0.14 & 0.052 & 0.049 \\
1 & 17.0 & 0.12 & 0.066 & 0.058 \\
\hline
\end{tabular}

observation is good, particularly as regards the dependence of thickness on rim shape.

\section{Evolution of the Bright Rims}

In order to give a qualitative picture of the evolution it is necessary to assume that inhomogeneities exist in the initial medium, with the density decreasing from the center of the inhomogenity toward its outside. The mechanism of evolution must be one which will be capable of amplifying the initial density inhomogeneities and of producing the observed shapes.

When the ionizing radiation first is incident on this region, the size of the rim or visible boundary of the region will be determined by the density at which the $R$-critical condition is reached. Since the $R$-critical condition is not a stable one, and the conditions which follow the $R$-critical condition require a higher density (which is found closer to the center of the region), the initial stage of the evolution is short lived and associated with the largest size of rim.

Consider what happens on a line connecting the source of radiation with the center of the region. The matter on the outer edge of the region nearer the star will be compressed toward the center until a $D$-critical density is reached, after which there will be no further large-scale motions of the gas. But on a line connecting the exciting star with points to the side of the center, the density of matter will not easily become great enough to effect the $D$-critical condition. Certainly at some distance from the center of the region, the density will never become great enough to allow a stable $D$-critical condition, and the matter at these points will remain in motion, with respect to the center of the region. This action can be thought of as an etching effect of the radiation which will produce an elongated shape pointing in the direction of the exciting radiation.

This agrees at least qualitatively with the observations. The rim is larger at the beginning of its evolution and smaller and elongated toward the exciting star in the later stages. The more elongated rims would then be closer to the exciting star because they are the older rims.

\title{
On the Stability of Ionization Fronts
}

\author{
F. D. KAHN \\ Department of Astronomy, Manchester University, Manchester, England
}

\section{INTRODUCTION}

$\mathrm{T}$ HE boundaries between HII and HI regions often have shapes which suggest that the motion of the interstellar gas is unstable there. A characteristic shape of this kind is the so-called "elephant's trunk," a long tongue of dark material (presumably an $\mathrm{HI}$ region) seen superimposed on the luminous HII region, and outlined by a thin rim brighter than the luminous background. A detailed study of these shapes has recently been made by Pottasch ${ }^{1}$; earlier observations had been made by Duncan ${ }^{2}$ and by Struve. ${ }^{3}$

Pottasch found that the bright rims always appear on the side facing towards the luminous star which is exciting the HII region, that these stars are almost always earlier in type than 09 , and that the density of

${ }^{1}$ S. Pottasch, Bull. Astron. Soc. Neth. 13, 77 (No. 471) (1956).

2 J. Duncan, Astrophys. J. 51, 4 (1920).

3 O. Struve, Astrophys. J. 85, 208 (1937). "the ionized gas in the rims is of the order 100 to 200 ions and electrons $/ \mathrm{cm}^{3}$. This means that the density, and consequently the gas pressure, in a bright rim is 10 or more times greater than it is in the background HII region. It seems unlikely that any instability can arise at such an HII-HI boundary with the hot and light gas in the HII region pushing against the cool and dense gas in the HI region. Van de Hulst ${ }^{4}$ and later Frieman ${ }^{5}$ have made some suggestions of what might happen if the hot gas were at higher pressure, and the latter concluded that elephants' trunks might, indeed, be formed. However, the relation between the gas pressures in the rims and the background HII regions indicates that it is more probable that the ionized gas is streaming away from the ionization front at the

${ }^{4}$ H. C. van de Hulst, Gas Dynamics of Cosmic Clouds, edited by H. C. van de Hulst and J. M. Burgers (North-Holland Publishing Company, Amsterdam, the Netherlands, 1955), Chap. 19.

5 E. A. Frieman, Astrophys. J. 120, 18 (1954). 
boundary of the HI region toward the exciting star, and that the background of ionized material is so rarefied that it can be treated to a good approximation as a vacuum. This picture resembles that of the rocket mechanism of Oort and Spitzer, ${ }^{6}$ which was also discussed by the author. ${ }^{7}$ In the following we investigate how and when this mechanism can lead to instabilities in the motion of the interstellar gas, and whether it is likely that the required conditions can be fulfilled. If they cannot, we must conclude that the elephants' trunks do not result from an instability, but reflect an uneven distribution of density in the interstellar material prior to its being ionized.

\section{MECHANICAL EFFECTS AT AN IONIZATION FRONT}

As first shown by Strömgren, ${ }^{8}$ the transition from almost complete ionization in a part of interstellar space to almost complete absence of it usually takes place within a very short distance. This has recently been confirmed by Goldsworthy's study ${ }^{9}$ of the motion of an ionization front. We idealize the problem and assume that the HII and HI regions are separated by a sharp ionization front (I front).

A number of relations have been deduced in an earlier paper, ${ }^{7}$ to which the reader is referred. Here we consider the following problem: At time $t$ let ionizing radiation of intensity $J_{F}$ photons $\mathrm{cm}^{-2} \mathrm{sec}^{-1}$ be incident on the I front from the direction of negative $x$. It is supposed that the state of the gas is $D$-critical (see $\mathrm{Kahn}^{7}$ or Pottasch ${ }^{1}$ for the terminology), and continually adjusts itself to this condition. It is further assumed that $J_{F}$ increases with time. The pressure $p_{F}$ in the HI region just ahead of the front is related to $J_{F}$ by the formula [see Kahn? ${ }^{7}$ Eq. (42)]

$$
p_{F}=4 m Q J_{F} / 5 \text {. }
$$

Here $m$ is the mass of a hydrogen atom and $\frac{1}{2} m Q^{2}$ is the excess above the Lyman limit of the average energy given to the gas per ionization, which is assumed to be constant-i.e., we assume that there is no change in spectral quality of the radiation. The pressure will therefore increase with $J_{F}$, and so will the sonic speed $c_{F}$ just ahead of the front. A compression wave is then sent into the quiet gas. The mean speed of advance of the front relative to the neutral gas is given by

$$
U=3 c_{F}^{2} / 4 Q \text {. }
$$

It is now supposed that the front has slight corrugations, so that at the ordinate $y$ it is displaced by a distance $\delta=\xi_{F}(t) \cos k y$. These corrugations cause corrugations in the pressure waves which are sent ahead. If the sonic speed at the front at time $t$ has the value $c_{F}(t)$, then the sonic speed at $t$ at the position

6 J. H. Oort and L. Spitzer, Astrophys. J. 121, 6 (1955).

7 F. D. Kahn, Bull. Astron. Soc. Neth. 12, 187 (No. 456) (1954).

8 B. Strömgren, Astrophys. J. 89, 526 (1939).

F. A. Goldsworthy, Communication to this Symposium (1957)
$x=x_{F}(t)+X$ and ordinate $y$ will have the value

$$
c_{F}\left(t-\frac{X-\delta}{c_{F}}\right)=c_{F}\left(t-X / c_{F}\right)+\sigma \delta .
$$

Here $\sigma=c_{F}^{-1} d c_{F} / d t$. We will be concerned with the variations of $c_{F}$, given by:

$$
C(t)=\sigma \delta=\sigma \xi_{F}(t) \cos k y .
$$

There will be a proportional pressure excess, and a motion will be set up in the neutral gas ahead of the front. Close to the front this motion will, for a monatomic gas, be governed by the equations:

$$
\frac{\partial^{2} \delta}{\partial t^{2}} \equiv \frac{\partial u}{\partial t}=-\frac{1}{\rho} \frac{\partial p}{\partial x}=-3 c \frac{\partial C}{\partial x} \approx-3 c_{0} \frac{\partial C}{\partial x},
$$

where $u, v$ are velocity components parallel to $O x$ and $O y$, and $c_{0}$ is an appropriate mean value for $c_{F}$. We seek solutions of the form:

$$
\begin{aligned}
\delta & =\xi_{F}(t) \cos k y=\xi_{0} e^{\mu t} e^{-n(x-U t)} \cos k y \\
C & =\sigma \xi_{0} e^{\mu t} e^{-n(x-U t)} \cos k y .
\end{aligned}
$$

Substitution into (5) immediately gives

$$
(\mu+n U)^{2}=3 \sigma c_{0} n \text {. }
$$

To the same approximation the equation of continuity becomes

$$
\begin{aligned}
\frac{\partial^{2} \rho}{\partial t^{2}} \approx-\rho\left(\frac{\partial^{2} u}{\partial x \partial t}+\frac{\partial^{2} v}{\partial y \partial t}\right) & \approx \frac{\partial^{2} p}{\partial x^{2}}+\frac{\partial^{2} p}{\partial y^{2}} \approx c_{0}^{2} \frac{\partial^{2} \rho}{\partial x^{2}}+\frac{\partial^{2} \rho}{\partial y^{2}} \\
w \frac{\partial^{2} C}{\partial t^{2}} & \approx c_{0}^{2}\left(\frac{\partial^{2} C}{\partial x^{2}}+\frac{\partial^{2} C}{\partial y^{2}}\right),
\end{aligned}
$$

from which we derive

$$
(\mu+n U)^{2}=c_{0}^{2}\left(n^{2}-k^{2}\right) .
$$

Hence

$$
n=\frac{1}{2}\left[\frac{3 \sigma}{c_{0}}+\left(\frac{9 \sigma^{2}}{c_{0}^{2}}+4 k^{2}\right)^{\frac{3}{3}}\right]
$$

(the positive root is necessary to make $\delta$ and $C$ vanish at infinity). The disturbance extends for an effective distance $1 / n$ into the neutral gas ahead; this effective distance is largest when $k=0$ and then equals $c_{0} / 3 \sigma$. The variation of $c$ itself within this distance will be small.

It follows from (7) that $\mu$ can be positive only if

$$
n<3 \sigma c_{0} / U^{2},
$$

which implies, with the aid of (9), that

$$
k<\left(3 \sigma c_{0} / U^{2}\right)\left(1-U^{2} / c_{0}^{2}\right)^{3} .
$$


The maximum instability occurs when

$$
k=k_{m}=\frac{3 \sigma c_{0}}{4 U^{2}}\left[1-\frac{U^{2}}{c_{0}^{2}}\right]^{\frac{1}{3}} ; n=n_{m}=\frac{3 \sigma c_{0}}{4 U^{2}} ; \mu=\mu_{m}=\frac{3 \sigma c_{0}}{4 U} .
$$

We may observe that in the case of a negative value of $\sigma$ (decreasing sonic speed at the front), the roots of (7) have the form

$$
\mu=-n U \pm i\left(-3 \sigma c_{0} n\right)^{\frac{1}{2}}
$$

and the corrugations give rise to exponentially damped waves traveling parallel to $O y$.

In terms of $Q$ and $c_{0}$ we have

$$
U=\frac{3 c_{0}^{2}}{4 Q} ; \quad k_{m}=\frac{4}{3} \frac{\sigma Q^{2}}{c_{0}^{3}}\left(1-\frac{9 c_{0}^{2}}{4}\right)^{\frac{1}{2}} ; \quad \mu_{m}=\frac{\sigma Q}{c_{0}} .
$$

To take some typical values: for an $\mathrm{O} 7$ star at $50000^{\circ} \mathrm{K}$ we have $Q=2.9 \times 10^{6} \mathrm{~cm} / \mathrm{sec}$; for atomic hydrogen at $100^{\circ} \mathrm{K}$ the sonic speed is $c_{0}=1.2 \times 10^{5} \mathrm{~cm} / \mathrm{sec}$. A typical value for $k_{m}$ is then $800 \sigma / c_{0}$. If we suppose that $J_{F}$ is multiplied by the factor $e$ in $10^{6}$ years, the relation .

$$
c_{F}^{5} \sim p_{F} \sim J_{F}
$$

gives $\sigma=\left(5 \times 10^{6} \text { years }\right)^{-1} /=6 \times 10^{-15} \mathrm{sec}^{-1}$. We then find that

$1 / \mu=210000$ years; $\lambda_{m}=2 \pi / k_{m}=3 \times 10^{16} \mathrm{~cm}=0.01 \mathrm{pc}$.

The smallest wavelength for which an instability occurs is $\frac{1}{4} \lambda_{m}$.

These values are very small compared with the dimensions of the elephants' trunks described by Pottasch. ${ }^{1}$ In order to make $\lambda_{m}=1 \mathrm{pc}$, with the given values of $\sigma$ and of the stellar temperature $T *$ determining $Q$, we should need $c_{0}=5.4 \times 10^{5} \mathrm{~cm} / \mathrm{sec}$, corresponding to a gas temperature of $2000^{\circ} \mathrm{K}$. The appropriate value of $\mu_{m}$ is now about 950000 years, so that the rate of growth of the trunks would be rather small.

\section{A REAPPRAISAL}

We have not yet considered the effect of any possible cooling of the ionized gas by $\mathrm{O}^{+}$ions. The importance of this process in HII regions has been emphasized by Spitzer ${ }^{10}$; it will lead quite rapidly to a cooling of the gas to about $8000^{\circ} \mathrm{K}$. Near an uncooled, $D$-critical front (compare $\mathrm{Kahn}^{7}$ for the terminology), the temperature on the ionized side is $T_{i, F}=(3 / 20) T_{*}$. If the exciting star is of type 07 , its temperature is $T_{*}=50000^{\circ} \mathrm{K}$ and $T_{i, F}=7500^{\circ} \mathrm{K}$; if the star is of type $05, T_{*}=80000^{\circ} \mathrm{K}$ and $T_{i, F}=12000^{\circ} \mathrm{K}$. The presence of the $\mathrm{O}^{+}$ions will make no difference in the first case; in the second they will produce a moderate amount of cooling. The ionized gas will then be raised only to about $8000^{\circ} \mathrm{K}$ and the I front will move as

\footnotetext{
${ }^{10}$ L. Spitzer, Astrophys. J. 120, 1 (1954).
}

though the exciting star has a surface temperature of $53000^{\circ} \mathrm{K}$. The motion is not changed in other ways and the results of Sec. 2 stand.

We might adopt the following picture of the development of bright rims in general. An $\mathrm{O}$ star begins to shine inside a cool interstellar cloud of low density. An I front of type R (see $\mathrm{Kahn}^{7}$ ) travels out through the cloud, leaving behind an HII region, until it meets a denser part of the cloud. A shock is then sent ahead, and the $I$ front behind the shock develops a rather denser ionized layer which is seen as a bright rim against the background HII region. If there is, at any time, an increase in the intensity of radiation reaching the I front, then an unstable motion should develop there. However, the wavelength of greatest instability turns out to be very small when reasonable assumptions are made for $T_{*}, T_{0}$, and the possible rate of increase in the intensity of the radiation. It seems that the appearance of the corrugations so produced would be quite different from that of elephants' trunks.

The result is rather unsatisfactory, for not only does the development of elephants' trunks remain unexplained, but the theory predicts the existence of smaller corrugations which are evidently absent. Only two conclusions are possible: the elephants' trunks are not due to an instability, but reflect an uneven distribution of the interstellar material before its ionization; and there must be some other reason why the instabilities of shorter wavelength are damped out.

\section{ABSORPTION IN THE BRIGHT RIM}

We therefore consider briefly the amount of absorption in the ionized layer behind the I front and ask what must be the rate of increase in the intensity $J_{0}$ of the ionizing radiation from the exciting star in order that $J_{F}$ shall remain constant. When the density of ions in the ionized layer is $n_{i}$, we may write

$$
\frac{\partial J}{\partial x}=-\beta n_{i}^{2},
$$

where $\beta$ is the recombination coefficient for ions and electrons, given by $\beta=3 \times 10^{-10} T^{-1} .^{11}$

To find $n_{i}$ and $T$ we consider the motion of the ionized gas. It will be assumed that this gas behaves as if the ratio of its specific heats had the value $\Gamma$ (we shall have $5 / 3 \geqslant \Gamma \geqslant 1)$. We introduce a coordinate $\eta$ measured from the front (positive in the direction to the non-ionized gas) and choose the origin of time at the instant when the motion began. In the ionized region the motion is in the direction of negative $\eta$. From the method of characteristics for one-dimensional flow (see, e.g., Pack $^{12}$ ), applied to the expansion

"C. W. Allen, Astrophysical Quantities (The Athlone Press, London, 1955), p. 89.

${ }_{12}$ D. C. Pack, Monthly Notices Roy. Astron. Soc. 113, 43 (1953) 
of the gas behind the ionization front we have

$$
c=c_{F}+\frac{\Gamma-1}{\Gamma+1} \frac{\eta}{t} ; \quad u=\frac{\eta}{t}-c
$$

since $u$ is equal to $-c$ at the front $\left(\right.$ see $\left.\mathrm{Kahn}^{7}\right)$. This result holds for

$$
0>\eta>-\frac{\Gamma+1}{\Gamma-1} c_{F} t ; \quad \text { where } \eta<-\frac{\Gamma+1}{\Gamma-1} c_{F} t,
$$

we have $c=0$.

Now

$$
\eta_{i} \sim \rho \sim c^{2 /(\Gamma-1)} \text { and } \quad T \sim c^{9} .
$$

Hence

$$
\beta n_{i}{ }^{2}=\beta_{F} n_{i F}{ }^{2}\left(c / c_{F}\right)^{(11-3 \Gamma) / 2(\Gamma-1)},
$$

and

$$
J_{0}-J_{F}=\int \beta n_{i}^{2} d \eta=\frac{2(\Gamma+1)}{9-\Gamma} B_{F} n_{i F}{ }^{2} c_{F} t .
$$

The dependence of $J_{0}-J_{F}$ on $\Gamma$ is weak: the limits of the fraction on the right-hand side are $8 / 11$ and $\frac{1}{2}$. For an $\mathrm{O} 7 \mathrm{star}, T_{*}=50000^{\circ} \mathrm{K} ; T_{i, F}=7500^{\circ} \mathrm{K} ; \beta_{F}=3.8$ $\times 10^{-3} \mathrm{~cm}^{3} / \mathrm{sec}$; for an O5 star, $T_{*}=80000^{\circ} \mathrm{K}: T_{i, F}$ $=8000^{\circ} \mathrm{K}$ (if the cooling by $\mathrm{O}$ ions is allowed for); and $\beta_{F}=3.5 \times 10^{-13} \mathrm{~cm}^{3} / \mathrm{sec}$.

The rate of increase in $J_{0}$ required to keep $J_{F}$ constant is least for $\Gamma=1$ when the gas remains isothermal. In this case:

$$
J_{0}-J_{F}=\frac{1}{2} B_{F}\left(\frac{2 J_{F}}{Q}\right)^{2} \frac{Q T}{2}
$$

(since $n_{i, F}=2 J_{F} / Q$ and $u_{i}=Q / 2=c_{F}$ ). On substitution of numerical values we have, for an $\mathrm{O} 7 \mathrm{star}$,

$$
J_{0}-J_{F}=1.3 \times 10^{-10} J_{F}^{2} t,
$$

or, with $J_{F}=1.5 \times 10^{9}$ photons $\mathrm{cm}^{-2} \mathrm{sec}^{-1}$, corresponding to $n_{i F}=10^{3}$ particles $/ \mathrm{cm}^{3}$,

$$
J_{0}=1.5 \times 10^{9}\left(1+2 \times 10^{-10} t\right) .
$$

For an $\mathrm{O} 5$ star we find substantially the same result. Thus $J_{0}$ must increase at the rate of 0.3 photon $\mathrm{cm}^{-2}$ $\mathrm{sec}^{-1}$ in order to keep $J_{F}$ constant. For comparison it can be seen from the data of Table II in $\mathrm{Kahn}^{7}$ that the value of $J_{0}$ is $1.9 \times 10^{10}$ photons $\mathrm{cm}^{-2} \mathrm{sec}^{-1}$ at $5 \mathrm{pc}$ distance from an $\mathrm{O} 7 \mathrm{star}$ in equilibrium, and is $4.8 \times 10^{11}$ photons $\mathrm{cm}^{-2} \mathrm{sec}^{-1}$ at the same distance from an $\mathrm{O} 5$ star in equilibrium. The required rate of increase in $J_{0}$ can be maintained at most for 2000 years, or for 50000 years, respectively. These are relatively short periods. Now $J_{F}$ must actually increase if there is to be any instability; it is seen that this cannot happen as a rule. If $J_{F}$ should increase at any time, it can do so only rather briefly. The work of Sec. 2 shows that only corrugations of very short wavelengths can then be amplified efficiently.

There is one final damping effect. If $\xi_{F}(t) \cos k y$ is the forward displacement of a corrugated I front at the ordinate $y$, the ionizing radiation must cross this additional distance through the bright rim there. It thus suffers an additional change in intensity, given by

$$
\Delta J=-4 \frac{\beta_{F} J_{F}^{2}}{Q^{2}} \xi_{F}(t) \cos k y .
$$

Since this gives a corresponding change in the sonic speed:

$$
\frac{\Delta c_{F}}{c_{F}}=\frac{1}{5} \frac{\Delta p}{p_{F}}=\frac{1}{5} \frac{\Delta J}{J_{F}},
$$

it is found that the destabilizing effect of the corrugated pressure waves will persist only if

$$
\sigma>\frac{4}{5} \frac{\beta_{F} J_{F}}{Q^{2}}=-\frac{2}{5} \beta_{F} n_{i F} \frac{c_{F}}{Q} .
$$

With our assumed values for $c_{F}, n_{i F}$, and $Q$ (in the case of an $\mathrm{O} 7 \mathrm{star}$ ), this leads to

$$
\sigma^{-1}<5300 \text { years, }
$$

meaning that $J_{F}$ must increase by a factor $e$ once in less than every 5300 years if there is to be any instability at all. This makes even larger the required rate of increase of $J_{0}$.

\section{CONCLUSION}

We have considered the stability, with respect to corrugations, of ionization fronts which are backed by a vacuum and which are advancing into a cold, nonionized gas. The only type found to be unstable is a $D$-critical I front at which the intensity of ionizing radiation is increasing. The instability is not likely to be important in practice, because of the strong damping effect due to the absorption of radiation in the bright rim following the front. The formation of elephants' trunks is therefore probably due to some other effect, possibly an unevenness in the distribution of neutral interstellar matter in the vicinity of $\mathrm{O}$ stars. $\mathrm{Münch}^{13}$ has independently found other reasons for believing that such an unevenness does occur.

\footnotetext{
${ }^{13}$ G. Münch, Communication to this Symposium (1957).
} 